\title{
Aproximaciones históricas a la constitución social del género. Una mirada a la escuela*
}

\author{
Claudia Mallarino Flórez ${ }^{* *}$
}

Recibido: marzo 19 de 2015 • Evaluado: abril 18 de 2015

Aceptado: Mayo 9 de 2015

\section{Resumen}

La modernidad posee formas singulares de discursividad somática que han constituido regímenes de saber/poder sobre el cuerpo generizado, socialmente situadas e históricamente determinadas. Las narrativas producidas socialmente frente a asuntos como la identidad, la sexualidad, los roles y el lugar de las feminidades y las masculinidades en las prácticas corporales, llevan a re-pensar algunas supuestas verdades con relación a la constitución social del género, en la comprensión de que tales significaciones no son universales o fijas, por el contrario, emergen en consonancia con los lugares, los tiempos y las circunstancias corporales en las que son producidas, pues acontecen en el cuerpo.

Palabras clave: cuerpo, género, sociedad, escuela, dispositivo, discursividades.

" Artículo de reflexión que presenta resultados derivados de la investigación realizada como tesis doctoral en el Doctorado Interinstitucional en Educación DIE - Universidad Pedagógica Nacional y Universidad del Valle, titulada: Cuerpos, sociedades e instituciones a partir de la última década del Siglo XX en Colombia., iniciada en febrero de 2012 y terminada en febrero de 2016.

"* Institución de procedencia: Universidad de San Buenaventura-Cali, Colombia. Nivel de formación: Candidata a doctora, Doctorado Interinstitucional en Educación DIE. Universidad Pedagógica Nacional y Universidad del Valle. Correo electrónico: cmallarino@ yahoo.es / mallarino.claudia@gmail.com 


\title{
Historical approaches of the social constitution of gender. A look at school
}

\begin{abstract}
Modernity has unique forms of somatic discursivities that have been constitute regimens of knowledge and power about the gendered body, social and historically situated. The socially produced narratives against issues such as identity, sexuality, roles and the place of the femininity and the masculinity in bodily practices, lead to re - think some supposed truths in relation to the social constitution of the genre, on the understanding that such meanings are not universal or fixed, on the contrary, emerge in line with places times and personal circumstances in where they are produced, as they occur in the body.
\end{abstract}

Keywords: body, genre, society, school, somatic discursivities. 


\section{Discursividades somáticas y regímenes de saber/ poder sobre el cuerpo generizado en la modernidad}

Las discursividades son enunciaciones sujetas a condiciones de posibilidad del saber acerca de algo que delimitan sus funciones merced al acto mismo de enunciar. Las discursividades producidas socialmente frente a asuntos como la identidad, la sexualidad, los roles y el lugar de las feminidades y masculinidades en las prácticas corporales, llevan a re-pensar algunas supuestas verdades con relación a la constitución social del género y la propuesta educativa que lo agencia dentro de la escuela, en la comprensión de que tales significaciones no son universales o fijas, por el contrario, emergen en consonancia con los lugares, los tiempos y las circunstancias corporales en las que son producidas, pues acontecen en el cuerpo.

Estas discursividades como enunciados de lo posible se articulan como un instrumento regulatorio para inscribir en los cuerpos realidades somáticas referidas al género y legitimadas en clave heteronormativa por la escuela, la familia, la religión, los medios, los libros y las conversaciones que sostenemos cotidianamente, al punto de que constituyen modos sociales aprobados o desaprobados de ser niñas y niños, jóvenes y adultos y de pensar la infancia, la juventud y la adultez. En este sentido decimos que la sociedad moderna posee formas singulares y específicas de discursividad que devienen regímenes de saber/poder o dispositivos para generizar el cuerpo. Michel Foucault propone el dispositivo como objeto de descripción del poder y lo presenta como "la red de relaciones que se pueden establecer entre elementos heterogéneos: discursos, instituciones, arquitectura, reglamentos, leyes, medidas administrativas, enunciados científicos, proposiciones filosóficas, morales, filantrópicas, lo dicho y lo no-dicho" (Castro, 2004, pp. 9899) y como la naturaleza del nexo que puede existir entre ellas. El dispositivo deviene agenciamiento colectivo de enunciación en donde los regímenes de signos, las máquinas de expresión (Lazzarato, 2006) actúan como ruedas del agenciamiento en razón de su "decibilidad" implícita.

Este modo de ser de la enunciación (decibilidad implícita), se logra en virtud de la "repetibilidad" de lo enunciado, pues a pesar de que emerge en diferentes ámbitos y se transforma, se mantiene. Tales decibilidades señalan la necesidad de pensarnos de conformidad con el ambiente del que hacemos parte y de acuerdo con los usos y costumbres de aquellos que nos 
precedieron, de tal manera que se moldea un cuerpo y se lo instala dentro de una cultura somática que lo hace portador de un género femenino o masculino y que lo identifica y lo autoriza para desempeñarse socialmente -roles- y actuar en consonancia -prácticas corporales-.

[...] siempre hago referencia a la construcción cultural de lo femenino. Pero el trasfondo es cómo de la historia de lo femenino y de la historia de lo masculino construimos nuestras identidades contemporáneas $[\ldots]$ me inspiro en Brigitte Bardot porque es un referente de la feminidad de mi época $[. .$.$] conozco sus películas, su entorno cinematográfico [...] Enton-$ ces yo me parezco es a la actriz que sale en sus películas no necesariamente a la Brigitte que es una persona de carne y hueso. (Baptiste, 2015)

La materialidad que se produce en esta repetibilidad del discurso le da permanencia al saber y le otorga su positividad empírica, lo que lo naturaliza $^{2}$ como régimen de verdad, como discursividad. Al respecto se puede decir que,

[...] el hecho de que ni la palabra homosexual ni la homosexualidad (entendida como identidad) existían antes del siglo XIX (la pre-modernidad), no significa que los actos sexuales entre hombres o entre mujeres no hayan existido o que personas no hayan sentido deseo, atracción y placer erótico hacia personas del mismo sexo. Los cánones de la Iglesia Católica, la ley civil, la literatura y los textos médicos pre-modernos incluyen innumerables referencias a actos de intimidad que incluyen órganos genitales, anos, manos, muslos, bocas y lenguas, realizados por hombres y mujeres, que horrorizaban e incitaban a las sociedades de esa época. (Goldberg, 1992, pp. 1-26)

Estos actos eran parte de una categoría social muy amplia, dice Goldberg, de una lista muy heterogénea de prácticas corporales que terminan nombrándose con una palabra que las reduce y las condena a una sola realidad semántica: sodomía. El agenciamiento que se instituye vía el dispositivo, es un engranaje que resulta de la afectación recíproca entre saber

1 Artículo publicado en El País, Sección Protagonistas el 22 de marzo de 2015

2 Naturalizar es hacer ver algo (objeto, práctica) como natural a fuerza de repetirlo y de volverlo hábito, habitual. 
y poder y de los efectos que esta reciprocidad produce [poder del saber: saber experto / saber del poder: poder ilustrado]. Las discursividades de las que hemos venido hablando están fechadas y situadas en tanto son social e históricamente situadas y queremos tratarlas dentro de regímenes específicos de saber/poder en la escuela moderna y a propósito de un asunto en particular: los saberes acerca del cuerpo en la perspectiva de una cultura de género. Frente a lo anterior dice Butler:

El género es el aparato a través del cual tiene lugar la producción y la normalización de lo masculino y lo femenino junto con las formas intersticiales hormonales, cromosómicas, psíquicas y performativas que el género asume. Asumir que el género implica única y exclusivamente la matriz de lo «masculino" y lo «femenino» es precisamente no comprender que la producción de la coherencia binaria es contingente, que tiene un coste, y que aquellas permutaciones del Género que no cuadran con el binario forman parte del Género tanto como su ejemplo más normativo. (Butler, 2006, p. 70)

Las narrativas referidas al "género" más que un saber son una voluntad de saber en tanto son producto de una actitud ilustrada propia de la modernidad, pero también porque son emergencia de dispositivos que naturalizan modos de saber hegemónicos acerca de mujeres y hombres. El género así puede asumirse como mecanismo que naturaliza estas dicotomías: masculino/ femenino - hombre/mujer - macho/hembra, a través de discursos médicos y saberes disciplinares especializados (higiene, urbanidad, educación física, psicología, pedagogía, puericultura, etc.), controlados por el poder administrativo y económico del Estado y tramitados por la escuela y la familia entre otras instituciones, para delinear un cuerpo ciudadano atendiendo a diferencias anatómicas, fisiológicas y de género (decibilidades que delimitan roles culturalmente instituidos) con el objetivo de establecer una clara división sexual del trabajo, en donde el hombre va a entenderse como el sujeto productivo y la mujer quedará encargada de la administración del hogar. La entrada del concepto de género al movimiento feminista, sobre el cual volveremos luego, le otorgará un escenario contundente de lucha tanto teórica como política:

El cuestionamiento a la fórmula biología es destino formó parte importante de un modelo teórico de explicación de las diferencias entre varones y mujeres y dio un sostenido empuje a las estrategias feministas a partir de 
los años '60 [...] Las voces de las mujeres lesbianas y también las voces de las mujeres negras, serán las primeras en denunciar a un feminismo que, tras la categoría Mujer, no reconoce la singularidad que asume la subordinación en virtud de la raza, la clase y/o la elección sexual. (Fernández, 2003, p. 138)

Como se ve, también las desnaturaliza y las deconstruye (a las dicotomías) ya que lejos de tener garantizada su verdad, se va enredando en las lógicas de un campo estratégico de fuerzas, distante y extraño en donde están tejidos cuerpos inmersos en juegos móviles y desiguales que actúan al margen de esa voluntad de saber siguiendo sus propias lógicas, las lógicas del poder (Mallarino, 2012). Es esta condición del saber/poder que preside lo legítimo/ ilegítimo y lo central/periférico lo que ha sido contexto propicio para el ejercicio de los llamados dispositivos. Un análisis crítico acerca de la naturaleza de la presencia corporal en la escuela se pregunta por las corporalidades que la habitan y por los desafíos que enfrenta al momento de pensar una educación para la sexualidad y el género en el contexto de la formación ciudadana y en consecuencia, es inminente leer desde esta perspectiva el andamiaje institucional que sostiene a la escuela. "Algunas personas me han preguntado para qué sirve incrementar las posibilidades del género. Generalmente contesto que la posibilidad no es un lujo; es tan crucial como el pan" (Butler, 2006, p. 51). Atendiendo a lo anterior, se intentará elaborar una aproximación a la configuración del cuerpo generizado en la escuela como institución social, desde algunos dispositivos de identidad delimitados socialmente, que le señalan al cuerpo sexuado roles culturales y prácticas singulares.

\section{Constitución del cuerpo generizado en la escuela: una mirada histórica}

La determinación de formas de ciudadanía y civilidad propias de la vida republicana instituyó modos burgueses (maneras, gestos, vestido, alimentación) y de utilitarismo bio-político (higienización del cuerpo, la casa, la ciudad y el territorio), como tecnologías urbanas de control del cuerpo sano, productivo, vigoroso y educado, hasta bien entrado el siglo XX. El Estado nacional apoyado por la familia y la escuela fue perfilando un proyecto 
civilizatorio de ciudadanía que definía las coordenadas de un cuerpo cívico femenino y masculino controlado y regulado desde los idearios de progreso, industrialización, moralidad y modernización del país. En atención a esto, los ciudadanos varones debían ser hombres blancos, educados, productivos, padres de familia, debían educar a sus hijos en una escuela higienizada y tener en casa a una mujer reproductiva, que cuidara los niños y que se encargara de la economía doméstica. Diseñar las formas de gobierno corporal de la escuela se reveló como campo de aplicación entre otras cosas, de las tecnologías disciplinarias creadas para el convento, el cuartel, la prisión y la fábrica (Foucault, 2002), que usadas para mapear e intervenir los cuerpos de mujeres y hombres, garantizaron el cumplimiento de la asignación generizada de responsabilidades en el ámbito de la vida laboral y productiva. Esto quiere decir, dispositivos de regulación y de homologación / singularización de los sujetos que se han ocupado de la materialización de pautas de conducta y de normalización tanto de cuerpos aprobados como de aquellos considerados desviados.

Tiene que existir la posibilidad de admitir y afirmar una serie de "materialidades" que correspondan al cuerpo [...] el carácter innegable de estas "materialidades" en modo alguno implica qué significa afirmarlas en realidad, qué matrices interpretativas condicionan, permiten y limitan esa afirmación necesaria. (Butler, 2002, p. 108)

Si pensamos en la preeminencia de la heterosexualidad, vemos que la preparación para la maternidad y la vigilancia de los cánones de belleza femenina -peso, vello corporal, menstruación, porcentajes de grasa, etc.-, han posibilitado la materialización de una identidad en donde se controla la sexualidad femenina como dispositivo de poder que desempodera a la mujer pues la ubica frente a una representación hegemónica. Otro tanto ha sucedido con los niños y los jóvenes sujetos a dispositivos de control que configuran identidades y racionalidades objetivas del ser hombre/macho y que los han comprometido con una performatividad de género que al tiempo que puede dotarlos de una identidad sobreestimada, puede hacer de ellos sujetos invisibles que no se reconocen en los estereotipos masculinos definidos por los cánones dominantes. Esta proclividad hacia el estereotipo binario se logra merced a patrones exteriores delineados minuciosamente en 
los momentos adecuados: comportamiento, género, edad, aspecto externo, estilo de vida, hábitos y costumbres

[...] es posible ver que la socialización tiende a efectuar una progresiva somatización de las relaciones de dominación sexual [...] concretada en una construcción social biologizada y que produce e impone a hombres y mujeres conjuntos diferentes de disposiciones con respecto a los juegos sociales considerados fundamentales. Por medio de la masculinización de los cuerpos masculinos y la feminización de los cuerpos femeninos se efectúa una somatización inconsciente de una construcción perdurable. (Palomar Verea, 2002, pp. 6-7)

En la escuela moderna de la primera mitad del siglo XX se instala la urdimbre social y cultural de la que dispone el aparato de Estado para llevar a cabo su encomienda como progenitor, cuidador y dador de posibilidades de vida digna, en el entendido de que es a esta institución, entre otras, a quien se le encomienda esta tarea y se le solicita que se ordene en consonancia con los cambiantes objetivos políticos y las estructuras sociales, de tal suerte que proporcione respuestas flexibles a las presiones económicas que proceden de agencias nacionales e internacionales (Martínez Boom, 2004, p. 15).

Vemos entonces que en este período histórico la construcción de una identidad homogénea a través de la articulación de políticas del cuerpo en el sistema educativo, se ve naturalizada en prácticas corporales singulares que se pueden reconocer como: la reticulación espacio-temporal minuciosa del trabajo somático que produce cuerpos dóciles y disciplinados, en el ámbito de la distribución generizada de responsabilidades sociales para cumplir con el proyecto ciudadano (varones que trabajan y fecundan y mujeres que dan a luz, amamantan y administran el hogar); la vestimenta como tecnología del cuerpo (uniformes en la escuela, el vestido de 15 años para las jovencitas, el pantalón, saco y corbata para los varones, etc.); la institucionalización de tecnologías de gobierno que introducen en la escuela prácticas de administración del saber determinadas por las lógicas del progreso y la industrialización; el desplazamiento del discurso de la espiritualidad al de la moralidad y la vida civilizada de la burguesía acordes con el imperativo social del cuerpo sano y productivo; y muchas otras.

Lo anterior ha significado la emergencia en la escuela de modos legítimos de saber y autoridad para orientar concepciones específicas de géneros 
y sexualidades que producen cuerpos generizados, sexuados y sexualizados, así como modos concretos de inclusión/exclusión social y ciudadana por medio de patrones genitalistas fuertemente naturalizados.

La construcción de un referente identitario de masculinidad / feminidad ha otorgado a la escuela potestad para determinar coincidencias y disidencias sexo-genéricas frente a los patrones de validez entronizados socialmente y en consecuencia, naturalizar prácticas corporales que responden a una verdad encarnada que se verá fuertemente cuestionada en los años posteriores. La segunda mitad del siglo XX, aun manteniendo dichas prácticas y discursividades, ha sido escenario de transformaciones profundas. Surgen otros modos de agenciamiento ${ }^{3}$ del saber acerca del cuerpo que apelan a la corporalidad como anclaje de la subjetividad para determinar la trama de las relaciones sociales y la realidad de los inacabables y a menudo contradictorios procesos del individuo, para comprender su mundo (Merleau-Ponty, 2003).

Hacia los años 60 emergen movimientos sociales con un carácter glo$\mathrm{bal}^{4}$ que empiezan a sentar la premisa de un cuerpo que no está dispuesto a ser la consecuencia de una normatividad sorda y ajena a sus necesidades y que se entiende diverso, múltiple y ciudadano del mundo. Estos movimientos fortalecen el feminismo y otras resistencias como la lucha contra la discriminación étnica, la ciencia, la tecnificación y el capitalismo, porque estos y en general el planteamiento de mundo vigente no dan cuenta de las necesidades humanas. La revolución sexual, que es uno de sus efectos, da origen a concepciones acerca de la vida de relación y del lugar que deben ocupar las personas en la sociedad que permiten, entre otras cosas, que las mujeres, en los 60, decidan sobre su sexualidad tomando "la píldora", gobiernen su cuerpo y empiecen a hacer parte de la vida pública y laboral. ${ }^{5}$ Lo anterior

3 Vamos a ver cómo el saber necesita ser agenciado, esta es la condición que lo hace posible. La condición del saber de ser agenciado es lo que configura una discursividad (régimen de verdad) en tanto emerge porque hay fuerzas que lo instituyen y que a la vez necesitan de él para expresarse.

4 Hipismo, contracultura, feminismo, movimiento obrero, sufragista, etc. Todos ellos movimientos de hombres y mujeres que empezaron a dejar oír sus voces frente a lo que estaba viviendo el mundo en su conjunto, y que fueron capaces de descentrar la mirada y hacer suya la carne de otros cuerpos sometidos, borrados y degradados. Los movimientos de mujeres se dieron inicialmente entre británicas y estadunidenses, luego escandinavas y holandesas y paulatinamente fueron entrando en nuestras latitudes.

5 En el curso de la década posterior, el mundo va a hacer resonancia del atrevimiento 
cambia por completo el panorama económico, político y productivo en las sociedades de la naciente globalización económica a la que da paso el fin de la bipolaridad geopolítica que había instituido la guerra fría. Las relaciones intra familiares mutan en razón de que la figura femenina deja de ser su eje de gravitación y por ende, se transforma la naturaleza del anclaje familiasociedad, pues se disloca el estatuto de responsabilidad sobre la vida de los niños y sobre la fecundidad en términos de responsabilidad con el cuerpo social, que se le había asignado a la mujer (Mallarino, 2013).

Las corporalidades jóvenes a su vez, se están ocupando de configurar otras identidades somáticas. Estos cuerpos son mutables, están siempre en proceso de construcción y adaptación a los nuevos principios y órdenes impuestos por la sociedad en la que viven evidenciados como tendencia, moda, estereotipo y estandarización somática. Estas voces narran los efectos derivados de la disolución de referencias y coordenadas para vivir una vida con sentido y de la necesidad de hacerse a un estilo propio mediante el cuerpo y sus superficies sirviéndose de las tecnologías de la apariencia corporal. Voces marginales, a su vez, empiezan a denunciar configuraciones somáticas asimétricas como la dominación de los hombres sobre las mujeres -sistema patriarcal-, que recurre sutilmente a diversos mecanismos institucionales e ideológicos para mantener esta primacía y opresión en prácticamente todas las esferas de la vida privada y pública. Estos serán los llamados movimientos feministas. En el contexto de la tercera ola feminista, hacia la última parte del siglo XX, estas mujeres dejan oír sus voces renovadas:

Si el sexo proporciona una suerte de sustrato físico sobre el cual puede injertarse cualquier identidad genérica, o si, por el contrario, el género hunde inexorablemente sus raíces en el sexo; si, en verdad, el cuerpo sexuado es un dato o si el significado de las propiedades físicas — de las diferencias anatómicas entre mujeres y hombres- es en sí mismo consecuencia de los procesos de constitución del género: he aquí los problemas que el feminismo contemporáneo ha puesto de relieve. Al distinguir entre sexo y género y politizar el espacio así delineado, los feminismos contemporáneos han dotado de profundidad y posibilidad histórica a la feminidad, en todas las dimensiones de este término. (Ergas, 1993, pp. 605-606)

femenino. Pro familia ofrece cursos de orientación sexual y reproductiva (1965), Pablo VI condena la contracepción (1968) y se admite el aborto en Estados Unidos (1973). 
Una de las realidades sociales por las que el feminismo ha peleado a brazo partido es la violencia contra la mujer, un fenómeno social tan antiguo como la sociedad misma. Su puesta en marcha como tema de debate universal ha permitido, "apenas, visualizar la punta del iceberg de la victimización femenina en el hogar que permanece oculta, invisibilizada tras la cortina de la vida privada, de la intimidad familiar, bajo el supuesto de no admitir la intromisión de ajenos" (Ayala \& Hernández, 2012, p. 1). Otra prueba de la relevancia que ha tenido la lucha feminista finisecular y del nuevo siglo se da en el ámbito de la industria pornográfica, una industria montada sobre la base de la explotación, en donde el espectador se sirve del cuerpo femenino para su goce. La mujer frente a esto, ha puesto en vigencia otros modos de lo explícito respecto de su sexualidad. En la industria pospornográfica, una industria emergente que se rebela contra las relaciones de fuerza, la retórica y las discursividades de la pornografía, cambian los usos de la imagen femenina. Bajo la posibilidad de pensar la verdadera existencia de "una penetración al cuerpo que no defina únicamente la propia continuidad del mismo, la herida y la violencia han sido llevados a la escena de lo pospornográfico como forma de encaminar a la imagen sexualmente explícita hacia una posible literalidad de la penetración del cuerpo" (Díaz, 2012, p. 11).

Hay otras realidades corporales que se han estado discutiendo y difundiendo en el Ciclo Rosa, una alianza entre academia, arte y activismo fundada para "promover el reconocimiento de derechos de sectores sociales que han sido marginados o excluidos de la condición de ciudadanía plena" (Serrano, 2006, p. 10): "yo no me sentía marica, esto que me invadía era distinto $[\ldots]$ no se trataba de mi orientación sexual, esto que sentía, tenía sus raíces en mi esencia" (Serrano, 2006, p. 11). Así se expresa un joven a quien el régimen de verdad escolar sobre su cuerpo, lo sitúa en un no lugar. Las categorías varón y mujer establecidas desde criterios anatómicos no son ni universales ni válidas para un sistema de clasificación de género. Las categorías de tercer sexo y tercer género se sitúan de manera antagónica o por lo menos contradictoria con el dimorfismo sexual (Garfinkel, 1967). La gente, dice Garfinkel, construye categorías no solamente sobre la base de un cuerpo natural sino también sobre la base de "genitales culturales", en donde los cuerpos dislocan las categorías de varón/mujer y masculino/ femenino. Sus identidades, roles y prácticas corporales se pueden situar, evidentemente, en más de dos tipos de personas (sexos), varón/mujer y en más 
de dos géneros: masculino/femenino. La propuesta de la dicotomía sexual y de género, de los roles asignados a hombres y a mujeres y de las prácticas corporales que aseguraron el proyecto civilizatorio de la modernidad, se topa, a estas alturas, con territorios corporales que aún están en proceso de descifrar. En tanto todas estas verdades somáticas devienen dispositivos de constitución del cuerpo generizado y toda esta variedad de cuerpos pasan inevitablemente por la escuela, parece pertinente hablar más bien de diversas escuelas, así como de diversos modos de escolarización, pues más allá de si ella es consciente de esto, esos cuerpos ya están allí. Habrá que ver si el cuerpo que hace posible la escuela logra dar cuenta de la diversidad de cuerpos que la habitan.

\section{Referencias}

Ayala S., L. \& Hernández M, K (2012). La violencia hacia la mujer. Antecedentes y aspectos teóricos. En J.C. Coll (Ed.). Contribuciones a las Ciencias Sociales. Recuperado de: http://www.eumed.net/rev/cccss/20/ashm.html

Butler, J. (2002). Cuerpos que importan, sobre los limites materiales y discursivos del sexo. Buenos Aires: Paidós.

Butler, J. (2006). Deshacer el género. Barcelona: Paidós Ibérica.

Castro, E. (2004). El vocabulario de Michel Foucault. Un recorrido alfabético por sus temas, conceptos y autores. Universidad Nacional de Quilmes. Recuperado de: http://es.scribd.com/doc/11414155/castro-edgardo-el-vocabulario-de-m foucault

Díaz Zepeda, A. (2013). Escatologías pospornográficas. Del menstrual porn al vomit gore. En E. Muñiz García \& M. List Reyes (Coords.). VI Congreso Internacional de Ciencias, Artes y Humanidades "El Cuerpo Descifrado". La ciencia y la tecnología en las prácticas corporales (pp. 572-585). México: UAM-X. 
Ergas, Y. (1993). El sujeto mujer. El feminismo de los años sesenta-ochenta. En G. Duby \& M. Perrot (Dir.). Historia de las mujeres en Occidente, T. 5. El siglo XX (pp. 593-620). Madrid: Taurus.

Fernández, J. (2003). Los cuerpos del feminismo. En D. Maffía (Comp.). Sexualidades migrantes. Género y transgénero (pp. 138-154). Colección Temas contemporáneos. Buenos Aires: Feminaria.

Foucault, M. (2002). Vigilar y castigar. Nacimiento de la prisión. Buenos Aires: Siglo XXI.

Garfinkel, H. (1967). Studies in Ethnomethodology. New Jersey, Prentice Hall.

Goldberg, J. (1992). Sodometries: Renaissance Texts, Modern Sexualities. Stanford: Stanford University.

Lazzarato, M. (2006). Politicas del acontecimiento. Buenos Aires: Tinta Limón.

Mallarino, C. (2013). Cuerpos sociales, cuerpos escolares: una historia de encuentros y desencuentros. En W. Moreno (Coord.). Revista Iberoamericana de Educación. "El cuerpo en la escuela", 62: 89-105.

Mallarino, C. (2012). Las discursividades del arte. Un lugar para el dislocamiento de los dispositivos escolares. En A. Martínez Boom (Comp.). Revista Colombiana de Educación. "Educación, subjetividad y estética", 63: 187-212.

Martínez Boom, A. (2004). De la escuela expansiva a la escuela competitiva: dos modos de modernización en América latina. Venezuela: Grupo Greco/ Universidad de los Andes; Colombia: Fundación Universitaria Luis Amigó; España: Anthropos.

Merleau-Ponty, M. (2003). Fenomenología de la percepción. New York: Routledge.

Palomar Verea, C. (2002). Pierre Bourdieu y los estudios de género: convergencias $y$ divergencias. Centro de estudios de género de la universidad de Guadalajara. Précis, En: Revista Universidad de Guadalajara (24).

Serrano, J.F. (2006). Otros cuerpos, otras sexualidades. Editor académico. Instituto Pensar, Bogotá. 